\title{
PADI4 regulates osteosarcoma proliferation primarily via Wnt/ $\beta$-catenin and MEK/ERK signaling pathway
}

\section{Jianping Guo}

Maternal and Child Health Care Hospital of Zibo

Lei Yin

Zibo Central Hospital

Xuezhong Zhang

Zibo Central Hospital

\section{Peng Su}

Zibo Central Hospital

Qiaoli Zhai ( $\nabla$ zbszxyyzql@163.com )

Zibo Central Hospital https://orcid.org/0000-0002-5570-6604

\section{Primary research}

Keywords: Peptidylarginine deiminase 4 (PADI4), osteosarcoma, proliferation, Wnt/ $\beta$-catenin, MEK/ERK

Posted Date: July 31st, 2020

DOl: https://doi.org/10.21203/rs.3.rs-30878/v2

License: (c) (i) This work is licensed under a Creative Commons Attribution 4.0 International License. Read Full License 


\section{Abstract}

Background: Peptidylarginine deiminase 4 (PADI4), an important modification enzyme of proteins, has received increased attention for its role in tumorigenesis of several human cancers. However, the effect of PADI4 on osteosarcoma remains largely unknown. Here, we evaluated the impact and mechanism of PADI4 on osteosarcoma proliferation.

Methods: Impact of PADI4 on proliferation of osteosarcoma cells is detected by the method of CCK8 and colony formation assay. Expression of PADI4 as well as Wnt/ $\beta$-catenin and MEK/ERK signaling markers after knocking down or ectopically expressing PADI4 or PADI4 inhibitor treatment is investigated by Western blot and RT-PCR. Then we investigated relevance of the expression level of the PADI4 in osteosarcoma samples and paired normal tissues by Western blot and RT-PCR. To further confirm whether PADI4 affects osteosarcoma tumorigenesis in vivo, we performed tumor formation experiments in nude mice.

Results: Firstly, ectopically expressing PADI4 showed positive regulation on colony formation capacity of osteosarcoma cells. Secondly, PADI4 stimulated Wnt/ $\beta$-catenin and MEK/ERK signaling in osteosarcoma cells. Thirdly, expression of PADI4 is higher in osteosarcoma samples compared with normal tissues. In vivo experiment also verified the positive effect of PADI4 on the growth of transplanted tumors in nude mice.

Conclusions: Taken together, our results revealed PADI4 promoted proliferation of osteosarcoma via Wnt/ $\beta$-catenin and MEK/ERK signaling pathway. This study may expand our understanding of osteosarcoma tumorigenesis and identify PADI4 as a potential target for diagnosis and treatment of osteosarcoma.

\section{Background}

Osteosarcoma is the most frequent type of bone malignant tumor, especially in children and adolescence[1]. It is derived from the interstitial cell line. The rapid growth of the tumor is due to the direct or indirect formation of tumor bone-like tissue and bone tissue through the cartilage stage[2]. Early diagnosis and timely therapy have improved the survival rate of the disease[3, 4]. However, osteosarcoma is still a disease with high mortality in children and adolescents, the underlying mechanisms for osteosarcoma especially regarding its tumorigenesis are in urgent need of research[5].

Peptidylarginine deiminase 4 (PADI4) has been identified as an important modification enzyme and can convert both arginine and monomethyl-arginine to citrulline[6]. In recent years PADI4 has received more and more attention for its essential role in gene regulation[7, 8]. An increasing amount of evidences indicate that PADI4 plays critical roles in the progression of many types of tumors, such as esophageal squamous cell carcinoma[9], glioblastoma multiforme[10], ovarian cancer[11] and so on. The main mechanism that PADI4 works is by altering the structure of the chromatin or the activity of the protein via changing the citrullination modification of histones or target proteins[12-15]. However, the effects of PADI4 on osteosarcoma progression remains largely unknown. 
In our present study, results showed that the PADI4 expression was up-regulated in osteosarcoma tissues compared to the matched adjacent tissues. Additionally, PADI4 accelerated the proliferation of human osteosarcoma cell lines U2OS and Saos-2 both in vitro and in a mouse xenograft model, while knockdown PADI4 with shPADI4 or inhibition of enzyme activity with PADI4 inhibitor Cl-amidine had the opposite effects. Furthermore, we also investigated the mechanism of PADI4 in osteosarcoma progression. Our results indicated PADI4 involved in osteosarcoma proliferation primarily via Wnt/ $\beta$ catenin and MEK/ERK signaling pathway.

\section{Materials And Methods}

\section{Osteosarcoma tissue samples}

All osteosarcoma tissues and adjacent tissues used in this study were obtained from patient surgeries at Zibo Central Hospital Affiliated to Shandong University, Shandong, P. R. China and were confirmed by pathological diagnose. This study was approved by the ethics committee of Zibo Central Hospital Affiliated to Shandong University and was performed according to the principles of the Declaration of Helsinki.

\section{Cell culture and transfection}

The human osteosarcoma cell lines U2OS and Saos-2 were obtained from the Chinese Academy of Sciences Committee Type Culture Collection cell bank (Shanghai, China). U2OS cells were cultured in 1640 medium (Gibco, USA) supplemented with $10 \%$ fetal bovine serum (FBS; Gibco, USA) at $37^{\circ} \mathrm{C}$ with atmosphere containing $5 \% \mathrm{CO}_{2}$. Saos-2 cells were cultured in McCoy's $5 \mathrm{~A}$ medium (Gibco, USA) supplemented with $10 \%$ fetal bovine serum (FBS; Gibco, USA) at $37^{\circ} \mathrm{C}$ with atmosphere containing $5 \%$ $\mathrm{CO}_{2}$.

Constructs of pcDNA3.1-HA-PADI4, pcDNA3.1-HA-PADI4-C645S, pLK0.1-PADI4-shRNA and their control vectors were kindly provided by National Laboratory of Medical Molecular Biology, Department of Biochemistry and Molecular Biology, Institute of Basic Medical Sciences, Chinese Academy of Medical Sciences and Peking Union Medical College, China. Cells was transfected with these constructs using Lipofectamine 3000 (Invitrogen) as per manufacturer's protocol.

\section{CCK8 method}

The proliferation of osteosarcoma cells was detected by Cell Counting Kit-8 (CCK-8; Dojindo, Tokyo, Japan) according to the manufacturer's protocol. Briefly, cells were seeded at a density of $1 \times 10^{3}$ cells per well into a 96 -well plate and the assay was conducted at $24 \mathrm{~h}$ intervals for 5 days. For detection, $10 \mu \mathrm{L}$ CCK8 solution was added to each well and cells were incubated at $37^{\circ} \mathrm{C}$ for $1 \sim 2 \mathrm{~h}$. The absorbance was measured at $450 \mathrm{~nm}$ with a microplate reader (Thermofisher, USA).

\section{Western blotting}


Whole cell lysate was prepared from cultured cells with RIPA buffer. After isolated by SDS-PAGE and transferred to polyvinylidene difluoride membranes囚proteins on the membranes were blocked with $5 \%$ skimmed milk for 1 hour, followed by incubated with primary antibodies (anti-PADI4, anti-ERK, antiphospho-ERK, anti-MEK, anti-phospho-MEK, anti- $\beta$-catenin, anti-active $\beta$-catenin, anti-H3-Cit, 1:1000; antiGAPDH, 1:3000, Cell Signaling Technology, Beverly, USA) at $4^{\circ} \mathrm{C}$ overnight. Wash the membranes with TBST and then incubate with anti-Mouse/Rabbit IgG HRP-conjugated secondary antibodies (1:5000) for $1 \mathrm{~h}$ at room temperature. Chemiluminescence emission was visualized using a Tanon 5200 chemiluminescence image analysis system (Tanon, China).

\section{Reverse Transcription-polymerase Chain Reaction (RT-PCR)}

Total RNA was extracted from cells using TRIZOL reagent (Invitrogen) and was subjected to reverse transcription using the PrimeScript 1st Strand cDNA Synthesis Kit (TaKaRa). SYBR Premix Ex Taq II (TaKaRa) was used to perform PCR amplification. The relative mRNA expression of PADI4 is normalized by GAPDH. Data represents the mean value \pm S.D from at least three independent experiments. Primer sequences used for amplification are listed as follow: (F; forward; R; reverse)

PADI4,F:CCCAAACAGGGGGTATCAGT;R:CCACGGACAGCCAGTCAGAA

AXIN2,F:GAAACAGCTCCAGAGAGAAATG;R:GCTCTCCAACTCCAGCTTCAG

CCND1,F:GAGGAGAACAAACAGATCATCC;R:GGTAGTAGGACAGGAAGTTGTT

DKK1,F:CACTGATGAGTACTGCGCTAGT;R:TCAGAAGACACACATATTCCAT

MYC,F:ACCACCAGCAGCGACTCTGAGG;R:TCCAGCAGAAGGTGATCCAGAC

GAPDH, F: GAAGGTGAAGGTCGGAGTC; R: GAAGATGGTGATGGGATTT

\section{Colony formation assay}

Cells $\left(1 \times 10^{3}\right.$ per well) were seeded in 6 -well plates and cultured in medium at $37^{\circ} \mathrm{C}$ with $5 \% \mathrm{CO}_{2}$ atmosphere until visible colonies were formed. The cells were washed with PBS for 3 times and then fixed with methanol. After staining the cells with crystal violet, the number of colonies were counted and analyzed.

\section{Animal Models}

Nude mice (age of 6 weeks), purchased from Shanghai SLAC Laboratory Animal Co. Ltd. (Shanghai, China) were subcutaneously injected with stable clones of cell suspensions containing the PADI4 lentiviral vector or the control vector. The tumor volumes were then measured and calculated every 7 days. All animal experiments were approved by the Animal Care and Use Committee of Shandong University. 


\section{Statistical analysis}

Statistical analysis was performed using GraphPad Prism. The results were presented as mean \pm standard deviation (SD). Student's t test or ANOVA was used to analyze the statistical significance. Survival data of patients with osteosarcoma was determined and analyzed by the Kaplan-Meier method and the log-rank test. $P$ values less than 0.05 indicated statistical significance.

\section{Results}

\section{PADI4 significantly promotes proliferation and colony formation of osteosarcoma cells in vitro}

To verify the impact of PADI4 activity on proliferation of osteosarcoma cells, we treated U2OS cells with PADI4 inhibitor Cl-amidine for different time and performed CCK-8 assay to detect the cell growth curve. Results showed that PADI4 inhibitor significantly suppressed the proliferation of U2OS cells (Figure 1A). Besides cell viability detection, we also performed colony formation to further assess the effect of PADI4 on proliferation of osteosarcoma cells. Compared with control group, the number of colonies significantly declined in the PADI4 inhibitor treatment group. Ectopically expressing PADI4 increased the colony formation capabilities of U2OS cells, while PADI4-C645S (loss of its deimination activity) had minor effect (Figure 1B, C). In order to further validate the function of PADI4, we also used another osteosarcoma cell line Saos-2 to perform the same experiments and the results showed similar trend (Figure 1D-F). Taken together, these results together suggest that PADI4 significantly promotes osteosarcoma cell proliferation in vitro.

\section{PADI4 regulates Wnt/ $\beta$-catenin and MEK/ERK signaling in osteosarcoma cells}

Both Wnt/ $\beta$-catenin and MEK/ERK signaling are important signaling pathways that driving tumorigenesis in different types of human cancers. We next investigated the impact of PADI4 on Wnt/ $\beta$-catenin and ERK/MEK signaling in U2OS and Saos-2 cells. As shown in Figure 2A, phosphorylation of MEK and phosphorylation of ERK, as well as active $\beta$-catenin, decreased following the PADI4 inhibitor treatment. To further confirm this result, we used constructs of PADI4-WT/C645S (loss of deimination activity) and PADI4 knockdown pLKO.1-PADI4-shRNA to detect their effects on Wnt/ $\beta$-catenin and MEK/ERK signaling. Results consistently showed that knockdown of PADI4 markedly inhibited phosphorylation of MEK, phosphorylation of ERK and active $\beta$-catenin in both U2OS and Saos-2 cells, while ectopically expressing wildtype PADI4 induced these markers. For the PADI4 mutation C645S which lost deimination activity, no significant change of these signaling markers was observed (Figure 2B, C). These results together revealed that PADI4 significantly stimulated Wnt/ $\beta$-catenin and MEK/ERK signaling in osteosarcoma cells.

Furthermore, we also performed qRT-PCR assay to demonstrate the effect of PADI4 inhibitor on gene expression downstream of the Wnt pathway via $\beta$-catenin in both U2OS and Saos-2 cells (Figure 2D, E). Results indicated that CCND1 and MYC, which have been identified as the major target genes of $\beta$ catenin, exhibited decreased mRNA expression after inhibition of PADI4 activity. Significantly, the effect 
of PADI4 inhibition on CCND1 was dramatic, with the relative expression level falling down to 0.002 . We also assessed the expression of DKK1, which takes the function to inhibit Wnt/ $\beta$-catenin signaling by promoting the endocytosis of Wnt receptors LRP5 and LRP6. Results showed the expression of DKK1 was up-regulated by $\mathrm{Cl}$-amidine, suggesting the suppression of Wnt/ $\beta$-catenin signaling. However, the expression of AXIN2, an inhibitor of the Wnt signaling pathway, which down-regulates $\beta$-catenin by altering its stability, was contrary to our expectations. In our experiment, AXIN2 mRNA decreased after Clamidine treatment, which is conflict with its reported function. This may need further investigated.

\section{Increased expression of PADI4 in patients with osteosarcoma}

We next investigated relevance of the expression level of the PADI4 in osteosarcoma samples and paired normal tissues by Western blot and RT-PCR. Results showed that protein level of PADI4 was up-regulated in osteosarcoma tissues compared with control tissues (Figure 3A, B). Relative mRNA expression level of PADI4 was also higher in osteosarcoma tissues compared with control tissues (Figure 3C), which suggested the potential carcinogenesis role of PADI4 in osteosarcoma. Then we analyzed the survival data of patients with osteosarcoma. We collected the data from ELISA assays of patients' blood samples and data was sorted from low to high according to the level of expression of PADI4 in each sample. Take the first 50\% for the low expression, the last 50\% for the high expression, and then draw KM plot. Results suggested that patients with higher PADI4 levels had poorer prognosis than those with lower PADI4 levels (Figure 3D). These results collectively indicated that increased expression of PADI4 was positively correlated with osteosarcoma development and prognosis.

\section{PADI4 promoted the in vivo growth of U2OS cancer xenografts}

To further confirm whether PADI4 affect osteosarcoma tumorigenesis in vivo, we performed tumor formation experiments in nude mice. Stable clones of cell suspensions containing the PADI4 lentiviral vector or the control vector were injected into nude mice. The tumor growth was determined after the osteosarcoma xenografts. Results showed that mice injected with the cell containing the PADI4 lentiviral vector exhibited remarkably increased volume and weight of the transplanted tumors in comparison with the control group (Figure 4A-C).

\section{Discussion}

PADI4, as an important PTM enzymes, has received more and more focus for the role in cell proliferation, differentiation, apoptosis, especially in the tumorigenesis[9]. However, the role of PADI4 in osteosarcoma progression remained largely unknown. In the present research, we demonstrated that PADI4 promoted osteosarcoma progression and PADI4 inhibitor significantly suppressed proliferation of osteosarcoma. Firstly, we explored the impact of PADI4 on proliferation and colony formation of osteosarcoma cells. CCK8 assay showed that PADI4 inhibitor treatment significantly downregulated U2OS and Saos-2 cell growth. According to colony formation experiment, Cl-amidine inhibited colony formation capabilities while ectopically expressing wildtype PADI4 promoted colony formation capabilities. Interestingly, PADI4 
mutation, loss of its deimination activity, showed no significant impact on colony formation capabilities, which indicated deimination activity of PADI4 is vital for regulation of OS cell proliferation.

Subsequently, we investigated the underlying mechanism of PADI4 in the regulation of OS cell proliferation. Wnt signaling pathway is an evolutionarily conserved signaling pathway that regulates cell growth, differentiation, apoptosis, and self-renewal. In the occurrence and development of many types of human cancers, Wnt signaling pathway is often abnormally activated and can cooperate with or antagonize other signaling pathways to regulate tumor proliferation[16]. The mark of Wnt signaling is accumulation of active $\beta$-catenin, which transfers in nucleus and acts as transcription factor to induce transcription of target genes involved in cell division[17]. MEK/ERK, an essential signaling pathway, modulates cell proliferation, differentiation and survival[18]. Activation of MEK/ERK signaling cascade regulates gene expression related with aberrant proliferation of cancer cells. Excessive activation of ERK has been identified in various human cancers, such as breast cancer[19], oral cancer[20], melanoma[21] and so on. Aberrations in components of MEK/ERK pathway could drive oncogenesis, which exist in approximately $30 \%$ of human cancers[21,22]. ERK is the terminal node of the pathway and is activated by MEK via phosphorylation at its Tyr and Thr regulatory sites. Phosphorylation of MEK and phosphorylation of ERK represent activity of MEK/ERK signaling[22]. For the importance of Wnt/ $\beta$-catenin and MEK/ERK signaling in cell proliferation, we assessed the expression of key markers related with these signaling pathways after treatment with PADI4 inhibitor, ectopically expressing PADI4 or knockdown of PADI4. Results showed that Cl-amidine markedly decreased the phosphorylation of both MEK and ERK, as well as active form of $\beta$-catenin. Similar function was observed after knockdown of PADI4. MEK/ERK and Wnt/ $\beta$-catenin signaling were markedly induced by ectopically expressing wildtype PADI4 and were unaffected by PADI4 mutation. So far, few reports have explored the impact of PADI4 on signaling pathways related with cell proliferation. We identified the regulation of Wnt/ $\beta$-catenin and MEK/ERK signaling by PADI4, which also verified the role of PADI4 in OS cell proliferation.

The expression of PADI4 in osteosarcoma samples and paired normal tissues also supported our hypothesis that PADI4 contributed to osteosarcoma tumorigenesis. We also verified the effect of PADI4 on osteosarcoma tumorigenesis in vivo. Many studies have explored the function of PADI4 as an oncogene in various cancers, such as esophageal squamous cell carcinoma[9], glioblastoma multiforme[10], ovarian cancer[11] and so on. Our research also indicated PADI4 could promote osteosarcoma tumorigenesis, at least partly via regulation of Wnt/ $\beta$-catenin and MEK/ERK signaling. Importantly, PADI4 inhibitors, such as $\mathrm{Cl}$-amidine showed significant effect on repressing osteosarcoma proliferation, which deserved to be studied further as a potential cancer suppressor on its biological functions and underlying mechanism.

\section{Conclusion}

Taken together, our results indicated PADI4 could promote proliferation of osteosarcoma via Wnt/ $\beta$ catenin and MEK/ERK signaling pathway. This study may expand our understanding of osteosarcoma tumorigenesis and identify PADI4 as a potential target for diagnosis and treatment of osteosarcoma. 


\section{Declarations}

Acknowledgements

We would like to express our gratitude to the researchers and study participants for their contributions.

\section{Authors' contributions}

JG and QZ designed research and analyzed the data; $L Y, X Z$ and $P S$ collected the resource and performed the experiments; All authors read and approved the final manuscript.

\section{Funding}

This work was supported by the Natural Science Foundation of Shandong Province (grant number ZR2018BC019).

\section{Availability of data and materials}

All data generated or analyzed during this study are included in this published article.

\section{Ethics approval and consent to participate}

All osteosarcoma tissues and adjacent tissues used in this study were obtained from patient surgeries at Zibo Central Hospital Affiliated to Shandong University, Shandong, P. R. China and were confirmed by pathological diagnose. This study was approved by the ethics committee of Zibo Central Hospital Affiliated to Shandong University and was performed according to the principles of the Declaration of Helsinki.

\section{Consent for publication}

Not applicable.

\section{Conflict of interest}

All authors state that they have no conflicts of interest.

\section{References}

1. Mirabello L, Troisi RJ, Savage SA: Osteosarcoma incidence and survival rates from 1973 to 2004: data from the Surveillance, Epidemiology, and End Results Program. Cancer 2009, 115(7):1531-1543.

2. Whelan JS, Davis LE: Osteosarcoma, Chondrosarcoma, and Chordoma. Journal of clinical oncology : official journal of the American Society of Clinical Oncology 2018, 36(2):188-193.

3. Lin YH, Jewell BE, Gingold J, Lu L, Zhao R, Wang LL, Lee DF: Osteosarcoma: Molecular Pathogenesis and iPSC Modeling. Trends in molecular medicine 2017, 23(8):737-755. 
4. Gianferante DM, Mirabello L, Savage SA: Germline and somatic genetics of osteosarcoma connecting aetiology, biology and therapy. Nature reviews Endocrinology 2017, 13(8):480-491.

5. Isakoff MS, Bielack SS, Meltzer P, Gorlick R: Osteosarcoma: Current Treatment and a Collaborative Pathway to Success. Journal of clinical oncology : official journal of the American Society of Clinical Oncology 2015, 33(27):3029-3035.

6. Nakashima K, Hagiwara T, Ishigami A, Nagata S, Asaga H, Kuramoto M, Senshu T, Yamada M: Molecular characterization of peptidylarginine deiminase in HL-60 cells induced by retinoic acid and 1alpha,25-dihydroxyvitamin D(3). J Biol Chem 1999, 274(39):27786-27792.

7. Cuthbert GL, Daujat S, Snowden AW, Erdjument-Bromage H, Hagiwara T, Yamada M, Schneider R, Gregory PD, Tempst P, Bannister AJ et al: Histone deimination antagonizes arginine methylation. Cell 2004, 118(5):545-553.

8. Wang Y, Wysocka J, Sayegh J, Lee YH, Perlin JR, Leonelli L, Sonbuchner LS, McDonald CH, Cook RG, Dou $Y$ et al: Human PAD4 regulates histone arginine methylation levels via demethylimination. Science 2004, 306(5694):279-283.

9. Liu C, Tang J, Li C, Pu G, Yang D, Chang X: PADI4 stimulates esophageal squamous cell carcinoma tumor growth and up-regulates CA9 expression. Mol Carcinog 2019, 58(1):66-75.

10. Kosgodage US, Uysal-Onganer P, MacLatchy A, Kraev I, Chatterton NP, Nicholas AP, Inal JM, Lange S: Peptidylarginine Deiminases Post-Translationally Deiminate Prohibitin and Modulate Extracellular Vesicle Release and MicroRNAs in Glioblastoma Multiforme. Int J Mol Sci 2018, 20(1).

11. Lee W, Ko SY, Mohamed MS, Kenny HA, Lengyel E, Naora H: Neutrophils facilitate ovarian cancer premetastatic niche formation in the omentum. The Journal of experimental medicine 2019, 216(1):176-194.

12. Li P, Wang D, Yao H, Doret P, Hao G, Shen Q, Qiu H, Zhang X, Wang Y, Chen G: Coordination of PAD4 and HDAC2 in the regulation of p53-target gene expression. Oncogene 2010, 29(21):3153-3162.

13. Li P, Hu J, Wang Y: Methods for analyzing histone citrullination in chromatin structure and gene regulation. Methods in molecular biology 2012, 809:473-488.

14. Tanikawa C, Espinosa M, Suzuki A, Masuda K, Yamamoto K, Tsuchiya E, Ueda K, Daigo Y, Nakamura $\mathrm{Y}$, Matsuda K: Regulation of histone modification and chromatin structure by the p53-PADI4 pathway. Nature communications 2012, 3:676.

15. Leshner M, Wang S, Lewis C, Zheng H, Chen XA, Santy L, Wang Y: PAD4 mediated histone hypercitrullination induces heterochromatin decondensation and chromatin unfolding to form neutrophil extracellular trap-like structures. Frontiers in immunology 2012, 3:307.

16. Chen Z, Pan T, Jiang D, Jin L, Geng Y, Feng X, Shen A, Zhang L: The IncRNA-GAS5/miR-221-3p/DKK2 Axis Modulates ABCB1-Mediated Adriamycin Resistance of Breast Cancer via the Wnt/beta-Catenin Signaling Pathway. Molecular therapy Nucleic acids 2020, 19:1434-1448.

17. Wang $L$, Zheng $C$, Wu X, Zhang $Y$, Yan S, Ruan L, Dai H: Circ-SOX4 promotes non-small cell lung cancer progression by activating the Wnt/beta-catenin pathway. Molecular oncology 2020. 
18. Bhagwat SV, McMillen WT, Cai S, Zhao B, Whitesell M, Shen W, Kindler L, Flack RS, Wu W, Anderson B et al: ERK Inhibitor LY3214996 Targets ERK Pathway-Driven Cancers: A Therapeutic Approach Toward Precision Medicine. Molecular cancer therapeutics 2020, 19(2):325-336.

19. Karamanou K, Franchi M, Onisto M, Passi A, Vynios DH, Brezillon S: Evaluation of lumican effects on morphology of invading breast cancer cells, expression of integrins and downstream signaling. The FEBS journal 2020.

20. Wang SH, Wu HC, Badrealam KF, Kuo YH, Chao YP, Hsu HH, Bau DT, Viswanadha VP, Chen YH, Lio PJ et al: Taiwanin E Induces Cell Cycle Arrest and Apoptosis in Arecoline/4-NQO-Induced Oral Cancer Cells Through Modulation of the ERK Signaling Pathway. Frontiers in oncology 2019, 9:1309.

21. Han YH, Mun JG, Jeon HD, Park J, Kee JY, Hong SH: Gomisin A ameliorates metastatic melanoma by inhibiting AMPK and ERK/JNK-mediated cell survival and metastatic phenotypes. Phytomedicine: international journal of phytotherapy and phytopharmacology 2019, 68:153147.

22. Guo YJ, Pan WW, Liu SB, Shen ZF, Xu Y, Hu LL: ERK/MAPK signalling pathway and tumorigenesis. Exp Ther Med 2020, 19(3):1997-2007.

\section{Figures}



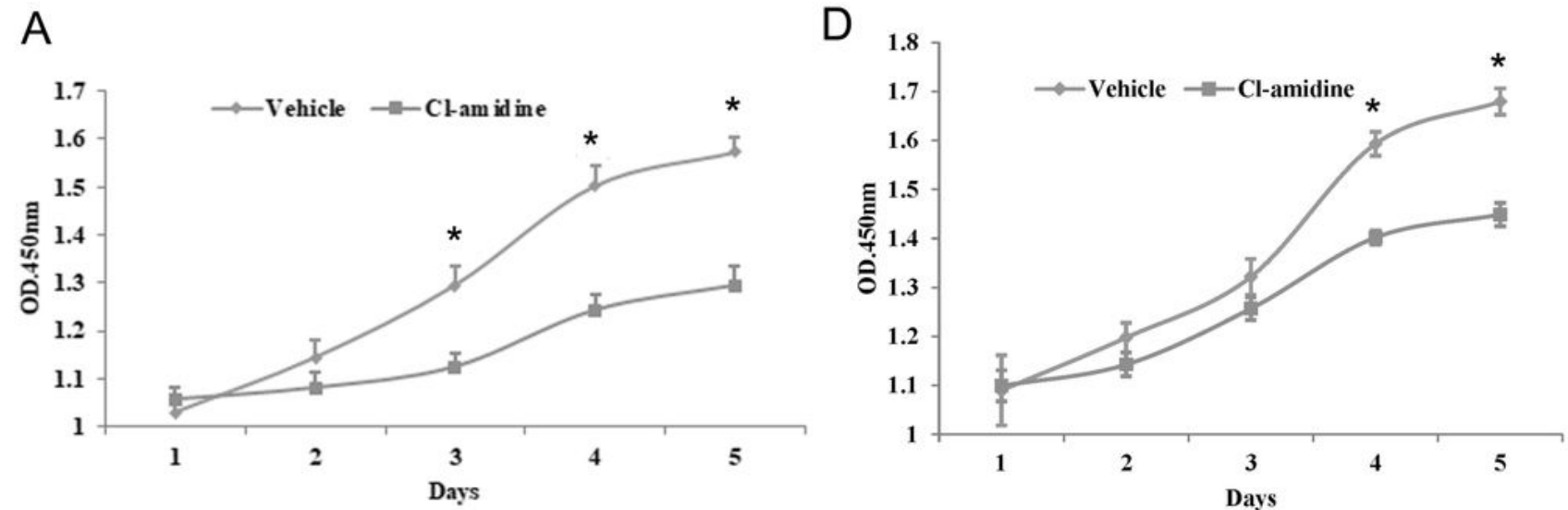

B

E
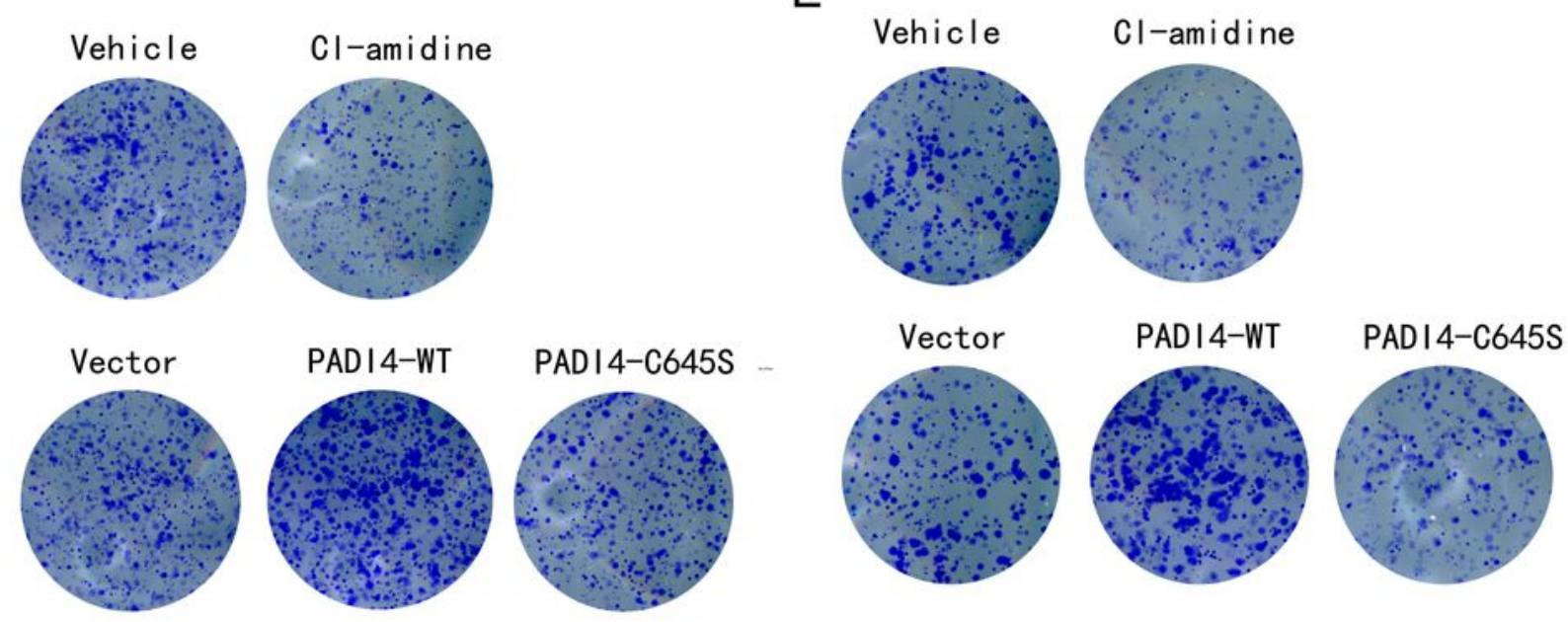

C

$\mathrm{F}$
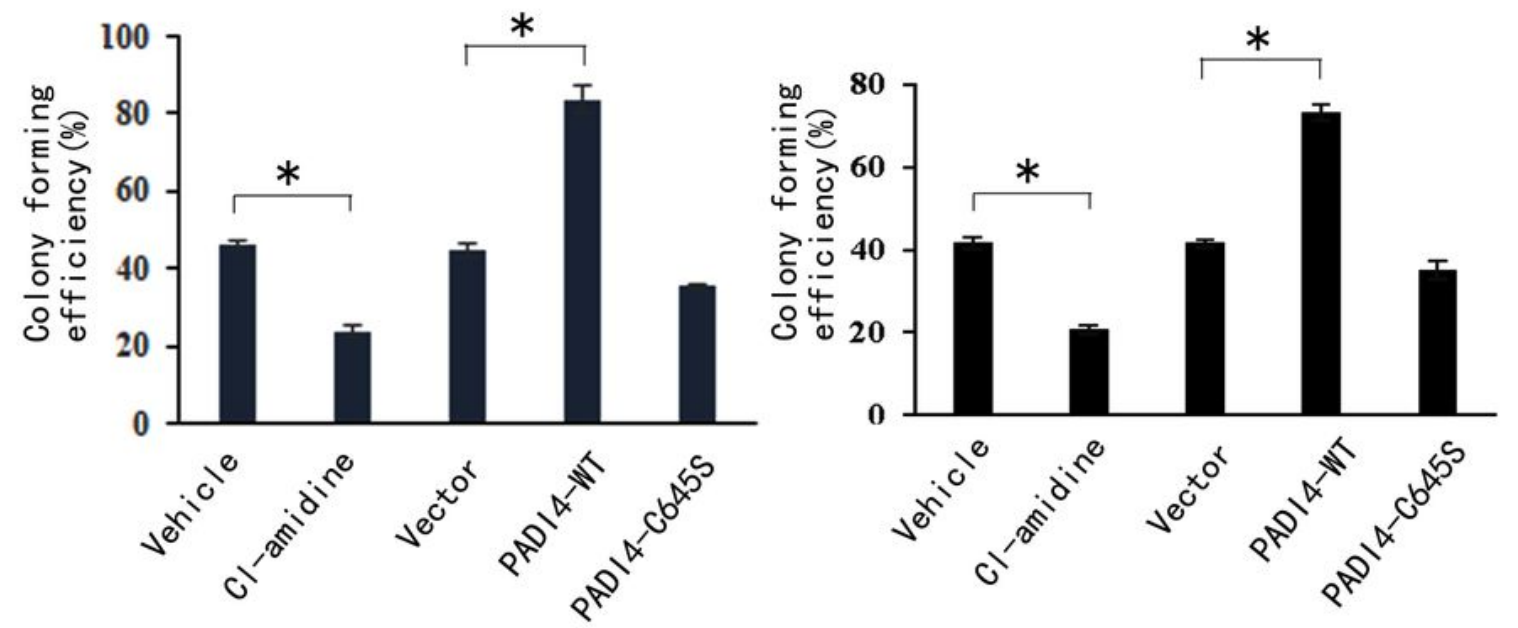

\section{Figure 1}

PADI4 promotes proliferation and colony formation of osteosarcoma cells. (A, D) CCK8 assay is performed for cell viability detection after PADI4 inhibitor $\mathrm{Cl}$-amidine treatment for indicated time in U2OS (A) and Saos-2 cells (D). (B, E) Colony forming assay is performed in U2OS (B) and Saos-2 cells (E) treated with PADI4 inhibitor or ectopically expressed with PADI4 plasmids. (C, F) The colony formation 
density was measured by ImageJ, and the colony formation efficiency (\%) is represented as bar graphs. Data are presented as the mean \pm SEM of three independent experiments $\left({ }^{*} P<0.05\right)$.

A

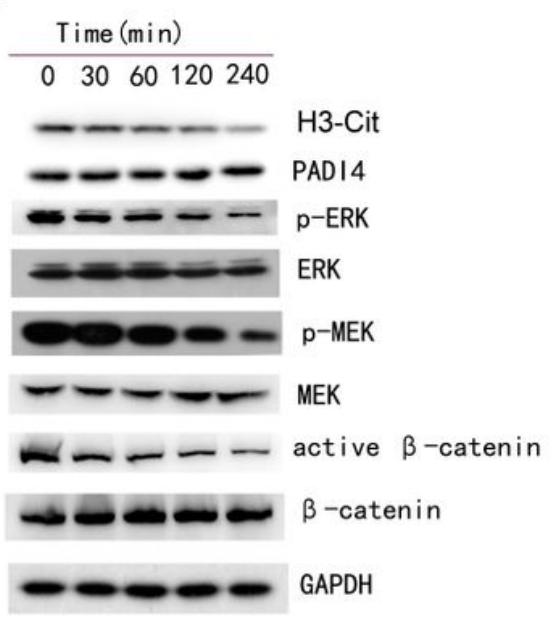

U2OS cells

D

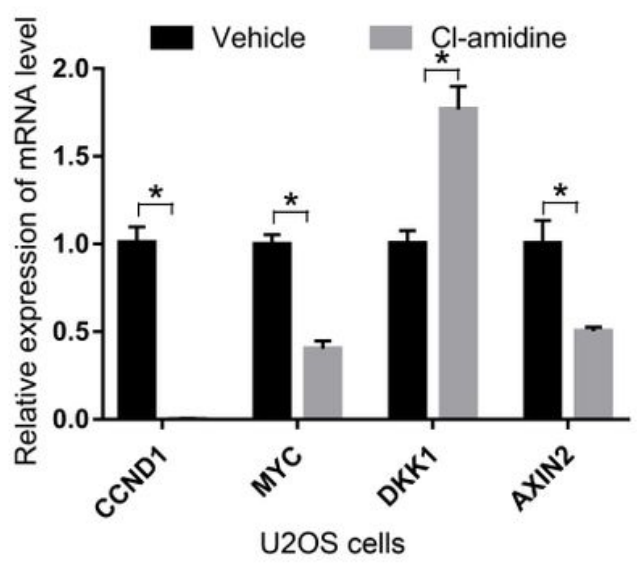

B

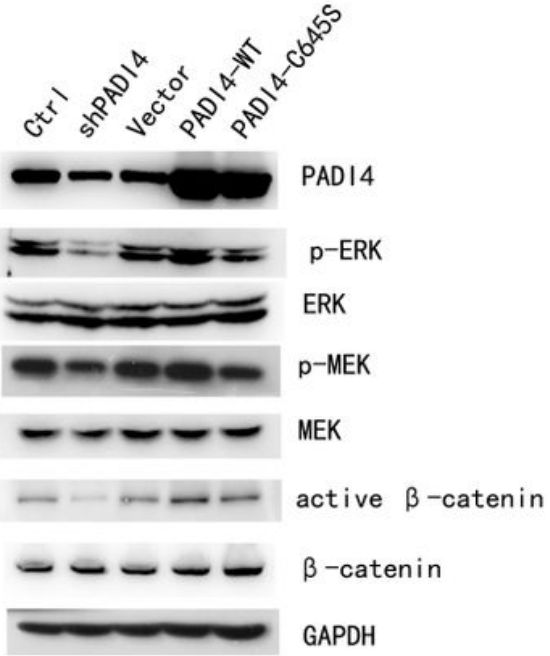

U2OS cells
C

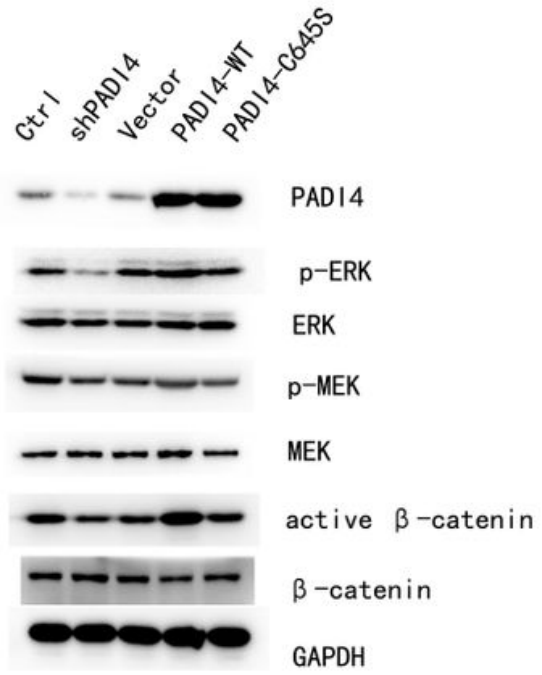

Saos-2 cells

E

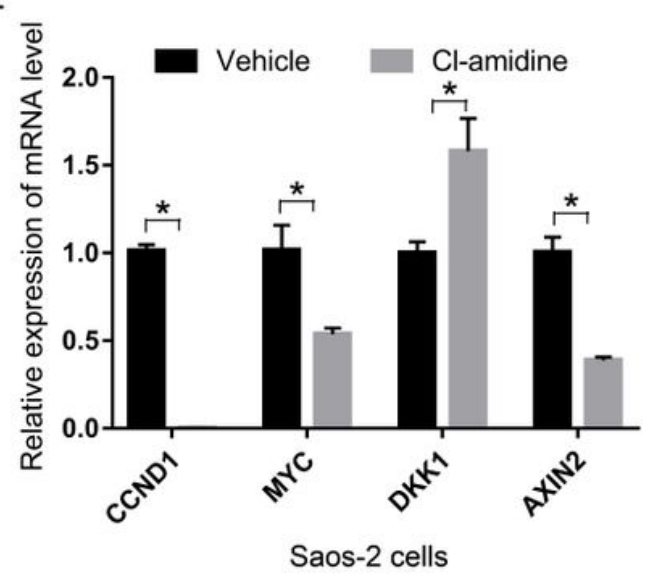

Figure 2

PADI4 regulates Wnt/ $\beta$-catenin and MEK/ERK signaling in osteosarcoma cells. (A) Western blot analysis of Wnt/ $\beta$-catenin and MEK/ERK signaling markers after $\mathrm{Cl}$-amidine treatment in U2OS cells for indicated time. GAPDH was used as a loading control. $(B, C)$ Expression of PADI4 as well as Wnt/ $\beta$-catenin and MEK/ERK signaling markers after knocking down or ectopically expressing PADI4 in U2OS (B) and Saos-2 (C) cells is investigated by Western blot. GAPDH was used as a loading control. (D, E) RT-PCR analysis of mRNA levels of Wnt/ $\beta$-catenin signaling markers after treatment with Cl-amidine in U2OS (D) and Saos-2 (E) cells. Data are presented as the mean \pm SEM $\left({ }^{*} \mathrm{P}<0.05\right)$. 
A

Adjacent Tissues Osteosarcoma Tissues

*1 *2 *3 *4 *5 *6 \#1 \#2 \#3 \#4 \#5 \#6

- - - - -

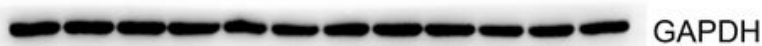

B

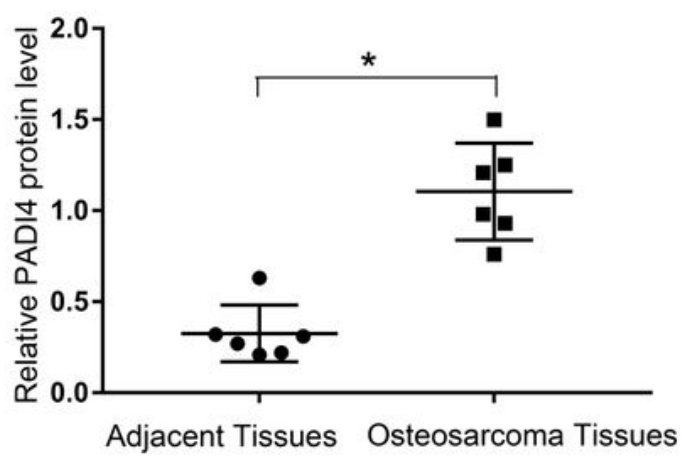

C

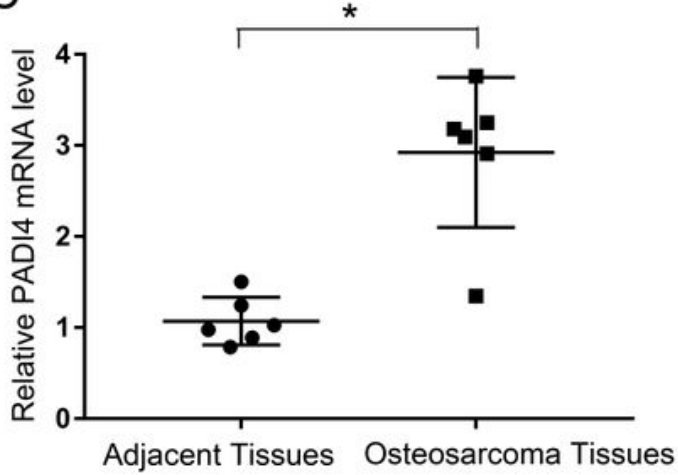

$\mathrm{D}$

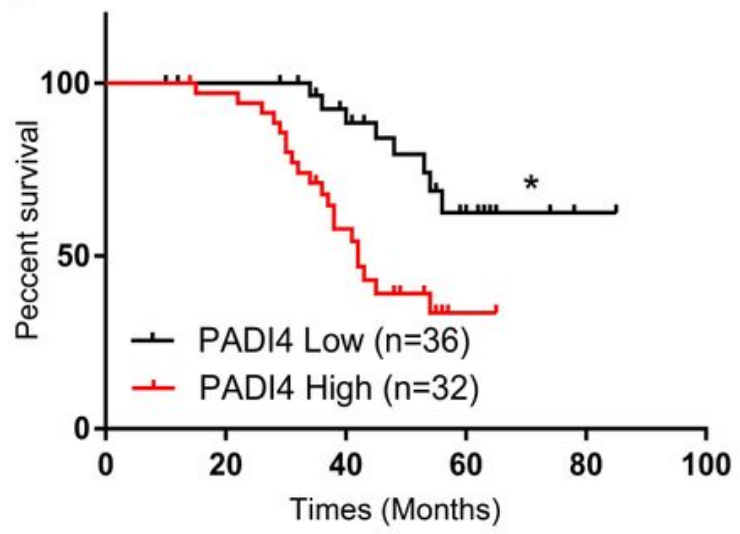

Figure 3

Increased expression of PADI4 in osteosarcoma tissues. (A) Expression levels of PADI4 in human osteosarcoma tissues and adjacent tissues is analyzed by Western blot. GAPDH was used as a loading control. (B) Quantification of protein levels. Data are presented as the mean $\pm S E M$. ${ }^{*} P<0.05$ vs. adjacent tissues. (C) RT-PCR analysis of mRNA levels of PADI4 in human osteosarcoma tissues and adjacent tissues. Data are presented as the mean \pm SEM. ${ }^{*} P<0.05$ vs. adjacent tissues. (D) Survival rate of PADI4 
high-expressed patients and PADI4 low-expressed patients by Kaplan-Meier survival analysis. ${ }^{*}<<0.05$ indicated statistical significance.

A

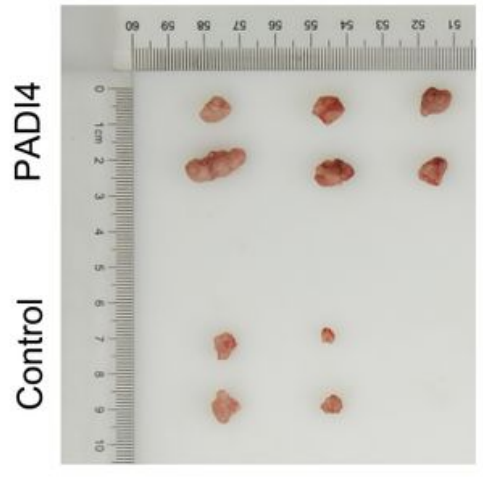

B

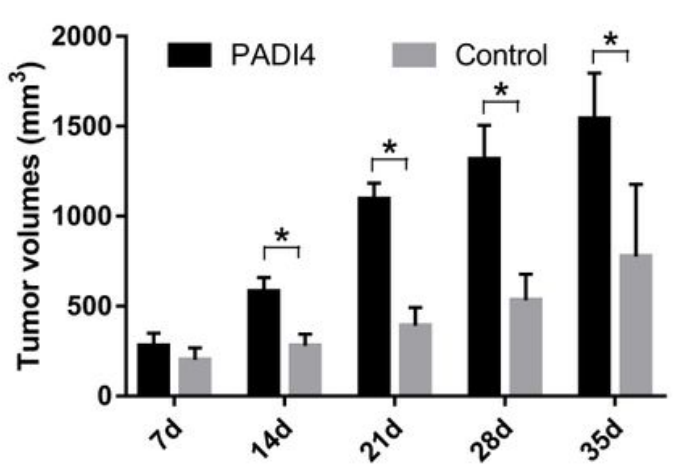

C

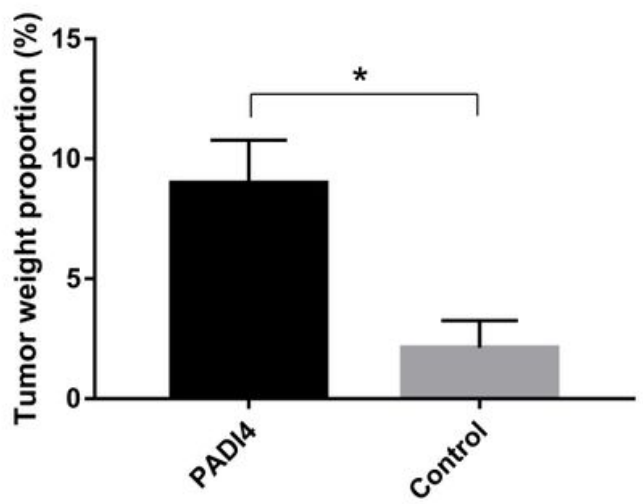

Figure 4

Overexpression of PADI4 enhances malignant growth of osteosarcoma cells in vivo. (A) Images of xenograft tumor formation in nude mice that were injected subcutaneously with cells expressing control or PADI4 lentiviral vector. (B) Quantification of tumor volume. (C) Quantification of tumor weight proportion (tumor weight/ total mouse weight). ${ }^{*} \mathrm{P}<0.05$ indicated statistical significance.

\section{Supplementary Files}

This is a list of supplementary files associated with this preprint. Click to download.

- FigS1.jpg 